\title{
Practical Approach To Reflux Symptoms
}

\author{
Dr Vikneswaran Namasivayam
}

\begin{abstract}
Reflux symptoms are commonly encountered in clinical practice. The vast majority of patients with typical reflux symptoms and no red flags may be placed on an empirical course of proton pump inhibitors (PPI). Patients with red flag findings require prompt further evaluation. Patients with symptoms not responding to PPI will require review of their compliance to PPI, clarification on which symptoms remain unresponsive and further evaluation in a directed manner.
\end{abstract}

Keywords: Gastroesophageal reflux disease; Proton pump inhibitors; Ambulatory esophageal pH monitoring; Refractory reflux symptoms;

SFP20I 7; 43(2): 48-5 I

\section{INTRODUCTION}

Gastroesophageal reflux disease (GERD) refers to the abnormal reflux of gastric contents that gives rise to chronic symptoms of heartburn, regurgitation, or injury to the oesophagus and contiguous organs of the upper aerodigestive tract. Symptoms suggestive of GERD are common and increasing in prevalence in Singapore. ${ }^{1}$ While the classic symptoms of GERD are usually readily recognised and satisfactorily treated with acid-suppressive medication, a substantial proportion of patients continue to have symptoms that are refractory to acid-suppressive medication and this is a common reason for a visit to a gastroenterologist. ${ }^{2}$ This review outlines a practical approach for patients who present with reflux symptoms and the further management of patients who have persistent symptoms that are unresponsive to acid-suppressive medication.

\section{ARE THE SYMPTOMS CONSISTENT WITH GASTROESOPHAGEAL REFLUX DISEASE?}

The classic symptoms of GERD are heartburn and acid regurgitation. Heartburn refers to the retrosternal burning sensation that rises towards the neck. Heartburn is relieved antacids and milk, and tends to occur after meals and upon lying down, typically at night. Regurgitation refers to the backflow of gastric contents, which are typically sour or bitter, into the chest or mouth.

\section{VIKNESWARAN NAMASIVAYAM}

Senior Consultant,

Department of Gastroenterology and Hepatology

Singapore General Hospital
Less common symptoms of GERD would include chest pain, dysphagia (sensation of food sticking in the chest), odynophagia (painful swallowing) and water brash (hypersalivation). These latter symptoms, though consistent with GERD, should prompt a careful assessment for an alternate cause - for example, cardiovascular disease in chest pain, oesophageal cancer in dysphagia and oesophageal ulcer in odynophagia.

Patients presenting with the following symptoms may require a more nuanced history taking to identify an alternate cause for their symptoms that require a different management strategy. Belching ("burping") refers to the retrograde passage of gas out of the mouth. It is a physiological phenomenon that allows for venting of the stomach when it is distended by swallowed gas after a meal. Belching may also occur in patients with GERD and functional dyspepsia. Patients who present with repetitive belching as an isolated complaint have an alternate mechanism for their belching, where air is sucked into the oesophagus and expelled without reaching the stomach. This is a condition known as supragastric belching. ${ }^{3}$ Patients are reassured of the benign nature of their belching, advised to avoid gum chewing, smoking, drinking carbonated beverages, and gulping their food and liquids. They may also be treated with diaphragmatic breathing (see below).

Bloating is often encountered in patients presenting to the primary care physician but it is not a symptom of GERD. In patients with bloating, any relation to meals or defaecation and a concomitant change in stool habits should be elicited to determine if the patient presenting with bloating has dyspepsia or irritable bowel syndrome. This would direct further evaluation and management.

\section{Any Red Flags to Warrant Early Endosocopy?}

Diagnostic tests are unnecessary in most patients with GERD who present with typical symptoms of heartburn and regurgitation. However, patients exhibiting red flags such as dysphagia, odynophagia, anorexia, weight loss, GI bleeding, or iron-deficiency anaemia should be referred for further GI evaluation. Patients with chest pain should have a cardiac evaluation before it is ascribed to GERD. Further investigations need to be performed in patients with red flags and those with symptoms that do not respond to PPI.

\section{Empirical Trial of Proton Pump Inhibitors}

Patients who present with typical symptoms of retrosternal heartburn and acid regurgitation in the absence of any red flags may be treated empirically with acid suppressive medication such as proton pump inhibitors (PPI). Complete resolution of typical symptoms with PPI would be a practical means of diagnosing GERD. As GERD is a chronic disease, symptoms may recur upon cessation of PPI and this would suggest a need for long-term management. 


\section{DEALING WITH THE PATIENT WITH REFLUX SYMPTOMS THAT ARE REFRACTORY TO ACID SUPPRESSION}

Patients with refractory reflux symptoms refers to those whose symptoms fail to respond partially or completely to a standard dose of PPI after a sufficient period of therapy, typically 8 weeks. ${ }^{4}$ A substantial proportion of patients with reflux symptoms continue to have symptoms that remain unresponsive to PPI. ${ }^{5,6}$

While PPIs are currently the most effective acid-suppressive therapy for GERD, we now recognise that PPIs vary in their efficacy for treating the individual manifestations of GERD. ${ }^{7,8}$ The response of a specific GERD manifestation is dependent on the degree to which that manifestation is related to acid. PPIs are most effective at achieving mucosal healing in patients who have oesophageal mucosal erosions on endoscopy, a subset of GERD known as erosive oesophagitis. However, the rate of complete symptom relief with a PPI is less than the rates of mucosal healing. Heartburn, the most characteristic reflux symptom, is the symptom that responds best to acid suppression. PPIs are less effective at treating regurgitation as PPIs increase the $\mathrm{pH}$ of gastric contents but do not eliminate the backflow of gastric juice that accounts for regurgitation.

We now also recognise that patients with persistent reflux symptoms refractory to PPI treatment often do not have GERD but are a heterogeneous group with a diverse range of conditions that can account for their symptoms (Table 1)., ${ }^{9,10}$ Further evaluation of the patient with refractory symptoms thus focuses on the following principal considerations.

1. Reviewing and reinforcing compliance to PPIs which are the most effective medication for GERD.

2. Carefully eliciting which symptom is not responding to a PPI as this may give clues to the underlying condition.

3. Evaluating for an alternate cause in a directed manner.

TABLE I: CAUSES OF REFRACTORY REFLUX SYMPTOMS.

\begin{tabular}{|l|}
\hline Non-GI diseases \\
\hline Heart disease \\
\hline Chest wall pain \\
\hline Non-oesophageal GI diseases \\
\hline Functional Dyspepsia \\
\hline Peptic ulcer disease \\
\hline Non-GERD oesophageal diseases \\
\hline Non-reflux Oesophagitis - pill injury, infectious oesophagitis, eosinophilic oesophagitis \\
\hline Achalasia and other oesophageal motility disorders \\
\hline Rumination syndrome \\
\hline Functional heartburn \\
\hline Oesophageal cancer \\
\hline Inadequate acid suppression \\
\hline Non-compliance - insufficient dose, dose timing \\
\hline
\end{tabular}

\section{PPI Compliance}

PPIs work by inhibiting the hydrogen-potassium ATPase pump in the gastric parietal cells. PPIs inhibit actively secreting proton pumps, hence they are most effective in reducing acid secretion when they are taken before meals, especially before breakfast. ${ }^{11}$ A large proportion of patients do not adhere to pre-meal dosing, hence optimising compliance should be undertaken to address an incomplete symptom response to a PPI. ${ }^{12}$ In patients who continue to be symptomatic, doubling the dose of PPI or switching PPIs has limited benefits. ${ }^{13}$ The addition of raft-forming agents (e.g. alginates) may improve heartburn in some patients. ${ }^{14}$

\section{Diagnostic Investigations in the PPI-refractory Patient}

Further evaluation of the patient with refractory reflux symptoms involves oesophagogastroduodenoscopy (OGD) and ambulatory $\mathrm{pH}$ testing and oesophageal manometry in selected instances.

\section{Oesophagogastroduodenoscopy}

Endoscopic examination of the upper GI tract is usually performed as the next step in evaluation. OGD allows for the detection of non-oesophageal conditions (e.g. peptic ulcers) which may present with reflux symptoms. OGD enables the detection of non-reflux oesophageal conditions such as drugs, infectious and eosinophilic oesophagitis, as well as oesophageal cancer. These conditions often have distinct endoscopic findings and diagnosis is confirmed on oesophageal biopsies. OGD also provides information on the severity of GERD (i.e. erosive oesophagitis) and the presence of complications such as Barrett oesophagus and oesophageal strictures. The presence of these findings would direct further management according to the etiology.

\section{Barium contrast studies}

Barium contrast studies are not recommended for the diagnosis of GERD as they have limited utility in detecting mucosal changes of GERD, and the demonstration of reflux on barium studies is not a reliable test for the diagnosis of GERD. It may be useful in selected patients for the demonstration of strictures and hiatal hernia, and in patients with suspected achalasia who are unwilling to undergo oesophageal manometry.

\section{Ambulatory oesophageal pH monitoring}

Ambulatory oesophageal $\mathrm{pH}$ monitoring provides a means to quantify acid exposure in the distal oesophagus as an objective measure of GERD. ${ }^{15}$ It entails placing a probe with a $\mathrm{pH}$ sensor through the patient's nose into the distal oesophagus. It transmits $\mathrm{pH}$ measurements from the distal oesophagus to a recorder worn on the person. These recorders have buttons that enable patients to record meals, symptoms events, and changes in posture. This recording is performed over a 24 -hour period. In patients who are unable to tolerate a nasal tube, wireless $\mathrm{pH}$ monitoring exists that entails attaching a $\mathrm{pH}$ capsule into the distal oesophagus during endoscopy. Generally, $\mathrm{pH}$ testing is performed when the patient is not taking any acid-suppressive medication.

The vast majority of patients with reflux symptoms will not require any $\mathrm{pH}$ testing. Testing is usually performed for preop- 
erative confirmation of GERD in patients being considered for fundoplication and in selected patients with refractory reflux symptoms. Ambulatory $\mathrm{pH}$ testing helps to determine if there increased amounts of acid exposure in the distal oesophagus which would be consistent with GERD - and whether there is a temporal correlation between the occurrence of symptoms and individual reflux episodes.

\section{Oesophageal manometry}

Oesophageal manometry entails placement of a catheter into the oesophagus to measure oesophageal peristalsis and lower oesophageal sphincter (LES) function. It is a test that enables the diagnosis of oesophageal motility disorders, especially achalasia. Oesophageal manometry is not indicated in the diagnosis of GERD. However, it is indicated in evaluating patients with dysphagia in the absence of a mechanical obstruction on OGD and in preoperative assessment of GERD patients considered for fundoplication to exclude achalasia. ${ }^{16}$ It is also performed in patients undergoing $\mathrm{pH}$ testing to determine the location of the LES that is used to guide the placement of the $\mathrm{pH}$ probe.

\section{PPI Refractory Manifestations}

A large proportion of patients with reflux symptoms that are unresponsive to PPI do not have GERD as the cause of their symptoms. ${ }^{10}$ While further evaluation is being carried out using a combination of the above investigations, careful history taking is required to clarify the complaints. Determining exactly which symptom remains unresponsive to PPI treatment may provide clues to the possible aetiology of the patient's symptoms and may be useful to direct further evaluation in a targeted manner.

\section{Persistent heartburn}

OGD is performed as the initial investigation in patients with persistent heartburn to exclude the conditions previously discussed. However, the majority of patients with reflux will have normal OGD. Patients with heartburn and normal OGD are a heterogeneous group of patients, some of whom may not have reflux at all as the cause of their symptoms. Further evaluation will need to be performed with $\mathrm{pH}$ testing. The results of $\mathrm{pH}$ testing help to differentiate patients with increased oesophageal acid exposure indicative of GERD from those with a completely normal study. Those with an elevated acid exposure may require escalation of acid suppression. The latter patients with a normal $\mathrm{pH}$ study do not have GERD but a condition known as functional heartburn. Functional heartburn is a condition characterised by persistent heartburn that is unresponsive to acid suppression and occurs in the absence of GERD, motility disorders, histopathologic mucosal abnormalities, major motor disorders, or structural explanations. ${ }^{17}$ The focus of management in functional heartburn is on reassurance and avoidance of repeated invasive testing. Treatment is empiric, with oesophageal pain modulators such as low-dose tricyclic antidepressants and selective serotonin reuptake inhibitors. Patients with functional heartburn should not undergo antireflux surgery as the outcome is likely to be poor.

\section{Persistent erosive oesophagitis}

Erosive oesophagitis refers to the presence of mucosal erosions in the oesophagus on endoscopy that would be consistent with GERD. Erosive oesophagitis heals well with acid suppression and is thus an uncommon finding in patients with reflux symptoms that have already received treatment with PPI prior to OGD. ${ }^{18}$ Hence the presence of erosive oesophagitis on OGD should prompt the following considerations. The patient's compliance to the PPI should be reviewed and reinforced. Alternate non-reflux aetiologies of oesophagitis such as medications (bisphosphonates, tetracyclines) and infections (cytomegalovirus, Candida, herpes) may need to be entertained in selected instances though most of these conditions have distinct endoscopic appearances that differentiate themselves from erosive esophagitis due to GERD. Acid suppression may need to be escalated and anti-reflux surgery (i.e. fundoplication) may be considered in selected instances of patients with erosive oesophagitis.

\section{Persistent regurgitation}

Regurgitation, as a symptom of GERD, is less responsive to acid-suppressive medication than heartburn. ${ }^{19}$ Fundoplication may be considered in selected GERD patients who have troublesome regurgitation. However, it is imperative that several conditions are excluded before fundoplication is undertaken to avoid an unsatisfactory outcome. An attempt must be made to determine whether the patient indeed has regurgitation or vomiting. The latter is characterised by the presence of nausea and forceful abdominal contractions which are absent in regurgitation. Vomiting entails an entirely different diagnostic work-up that encompasses gastrointestinal, intra-cranial and systemic diseases. In practice, the distinction between vomiting and regurgitation may not always be easily made and a broader list of differential diagnoses needs to be entertained that encompasses both symptoms in some patients.

In patients deemed to have regurgitation one has to exclude achalasia and rumination syndrome before fundoplication is considered. Achalasia is an uncommon disease characterised by absent oesophageal peristalsis and failure of relaxation of the LES. Classic symptoms include dysphagia, regurgitation, chest pain and weight loss. The regurgitation of bland retained oesophageal contents in achalasia may in some instances differentiate it from the sour acid regurgitation in GERD. Achalasia is diagnosed on oesophageal manometry. In patients with equivocal findings, barium contrast studies may be required as well to diagnose achalasia. OGD is performed in every patient with achalasia to exclude gastroesophageal cancers and other conditions that may mimic achalasia (i.e. pseudoachalasia). Treatment of achalasia is directed at mechanical disruption of the LES either by pneumatic dilatation or myotomy. The latter may be performed surgically or endoscopically. ${ }^{20}$

Rumination syndrome is a benign condition characterised by effortless regurgitation of recently ingested food into the mouth followed by remastication and swallowing back or spitting out. The regurgitant consists of recently swallowed food that can still be recognised by the patient and regurgitation typically occurs in the context of a meal. It does not affect sleep, unlike regurgitation in GERD which may occur as a nocturnal event affecting sleep. Rumination syndrome can affect adults of both gender across the age spectrum and is often misdiagnosed due to a lack of awareness of this benign condition. ${ }^{21}$ Patients are managed by reassurance, explanation of the condition and behavioral therapy which focuses on learning to recognise when 
rumination occurs and habit reversal with diaphragmatic breathing techniques. ${ }^{22}$ Rumination involves contraction of the abdominal wall muscles. Diaphragmatic breathing entails breathing in and out with the abdominal muscles which is incompatible with the abdominal wall contraction required for rumination to occur. Consistent practice of diaphragmatic breathing thus counters the act of rumination.

\section{CONCLUSION}

This review outlines a practical approach to the patient with reflux symptoms. The majority of patients with typical reflux symptoms and no red flags may be placed on an empirical course of proton pump inhibitors (PPI). Patients with red flag findings require prompt further evaluation. Patients with symptoms not responding to PPI will require further evaluation which would encompass a review of their compliance to PPI, clarification on which symptoms remain unresponsive, and further evaluation in a directed manner.

\section{REFERENCES}

I. Lim SL, Goh WT, Lee JM, Ng TP, Ho KY, Community Medicine GI Study Group. Changing prevalence of gastroesophageal reflux with changing time: longitudinal study in an Asian population. J

Gastroenterol Hepatol. 2005;20:995-100I.

2. El-Serag H, Becher A, Jones R. Systematic review: persistent reflux symptoms on proton pump inhibitor therapy in primary care and community studies. Aliment Pharmacol Ther. 2010;32:720-37.

3. Bredenoord AJ. Management of belching, hiccups, and aerophagia. Clin Gastroenterol Hepatol. 2013; I I ( I):6-12.

4. Fock KM, Talley N, Goh KL, Sugano K, Katelaris P, Holtmann G, et al. Asia-Pacific consensus on the management of gastro-oesophageal reflux disease: an update focusing on refractory reflux disease and Barrett's oesophagus. Gut. 2016;65: I402-15.

5. Niu XP, Yu BP, Wang YD, Han Z, Liu SF, He CY, et al. Risk factors for proton pump inhibitor refractoriness in Chinese patients with non-erosive reflux disease. World J Gastroenterol. 2013;19:3 I24-9.

6. Lee ES, Kim N, Lee SH, Park YS, Kim JW, Jeong SH, et al. Comparison of risk factors and clinical responses to proton pump inhibitors in patients with erosive oesophagitis and non-erosive reflux disease. Aliment Pharmacol Ther. 2009;30:154-64.

7. Kahrilas PJ, Howden CW, Hughes N. Response of regurgitation to proton pump inhibitor therapy in clinical trials of gastroesophageal reflux disease. Am J Gastroenterol. 20 I ; 106:1419-25; quiz 26.

8. Kahrilas PJ, Hughes N, Howden CW. Response of unexplained chest pain to proton pump inhibitor treatment in patients with and without objective evidence of gastro-oesophageal reflux disease. Gut. 20II;60:1473-8.
9. Roman S, Keefer L, Imam H, Korrapati P, Mogni B, Eident K, et al. Majority of symptoms in esophageal reflux PPI non-responders are not related to reflux. Neurogastroenterol Motil. 2015;27:1667-74. 10. Herregods TV, Troelstra M, Weijenborg PW, Bredenoord AJ, Smout AJ. Patients with refractory reflux symptoms often do not have GERD. Neurogastroenterol Motil. 2015;27:I 267-73.

I I. Hatlebakk JG, Katz PO, Camacho-Lobato L, Castell DO. Proton pump inhibitors: better acid suppression when taken before a meal than without a meal. Aliment Pharmacol Ther. 2000; I4: 1267-72. 12. Gunaratnam NT, Jessup TP, Inadomi J, Lascewski DP. Sub-optimal proton pump inhibitor dosing is prevalent in patients with poorly controlled gastro-oesophageal reflux disease. Aliment Pharmacol Ther. 2006;23:1473-7.

13. Fass R, Sontag SJ, Traxler B, Sostek M. Treatment of patients with persistent heartburn symptoms: a double-blind, randomized trial. Clin Gastroenterol Hepatol. 2006;4:50-6.

14. Manabe N, Haruma K, Ito M, Takahashi N, Takasugi H, Wada Y, et al. Efficacy of adding sodium alginate to omeprazole in patients with nonerosive reflux disease: a randomized clinical trial. Dis Esophagus. 20I2;25:373-80.

I5. Hirano I, Richter JE, Practice Parameters Committee of the American College of Gastroenterology. ACG practice guidelines: esophageal reflux testing. Am J Gastroenterol. 2007; 102:668-85. 16. Kahrilas PJ, Shaheen NJ, Vaezi MF, Hiltz SW, Black E, Modlin IM, et al. American Gastroenterological Association Medical Position Statement on the management of gastroesophageal reflux disease. Gastroenterology. 2008; I35:1383-91, I391.el-5.

17. Aziz Q, Fass R, Gyawali CP, Miwa H, Pandolfino JE, Zerbib F. Functional esophageal disorders. Gastroenterology. 2016; doi: 10.1053/j.gastro.2016.02.012. [Epub ahead of print].

18. Poh CH, Gasiorowska A, Navarro-Rodriguez T, Willis MR, Hargadon D, Noelck N, et al. Upper Gl tract findings in patients with heartburn in whom proton pump inhibitor treatment failed versus those not receiving antireflux treatment. Gastrointest Endosc. 2010;71:28-34.

19. Kahrilas PJ, Jonsson A, Denison H, Wernersson B, Hughes N, Howden CW. Regurgitation is less responsive to acid suppression than heartburn in patients with gastroesophageal reflux disease. Clin Gastroenterol Hepatol. 2012;10:612-9.

20. Marano L, Pallabazzer G, Solito B, Santi S, Pigazzi A, De Luca R, et al. Surgery or Peroral esophageal myotomy for achalasia: a systematic review and meta-analysis. Medicine (Baltimore). 2016;95:e300I.

2I. Absah I, Rishi A, Talley NJ, Katzka D, Halland M. Rumination syndrome: pathophysiology, diagnosis, and treatment.

Neurogastroenterol Motil. 2016;1-8.

22. Halland M, Parthasarathy G, Bharucha AE, Katzka DA.

Diaphragmatic breathing for rumination syndrome: efficacy and mechanisms of action. Neurogastroenterol Motil. 2016;28:384-91. 